\title{
ANTICANCER EFFECT OF PROBIOTIC SACCHAROMYCES BOULARDII SUPERNATANT ON HUMAN CACO-2 CELLS; AN IN VITRO STUDY
}

\author{
Samaneh Allahyari ${ }^{1}$, Shaghayegh Pishkhan Dibazar ${ }^{2}$, Babak Pakbin ${ }^{3}$, Razzagh Mahmoudi ${ }^{*}$, \\ Alireza Farasat ${ }^{5}$, Amir Peymani ${ }^{4}$, Peyman Ghajarbeygi ${ }^{6}$ and Nematollah Gheibi ${ }^{5}$ \\ ${ }^{I}$ Department of Food Hygiene and Safety, School of Health, Qazvin University of Medical sciences, Qazvin, Iran. \\ ${ }^{2}$ Department of Immunology, Faculty of Medical Science, Tarbiat Modares University, Tehran, Iran. \\ ${ }^{3}$ Faculty of Veterinary Medicine, Department of Food Hygiene and Quality of Control, University of \\ Tehran, Tehran, Iran. \\ ${ }^{4}$ Medical Microbiology Research Center, Qazvin University of Medical Sciences, Qazvin, Iran. \\ ${ }^{5}$ Department of Medical Biotechnology, Qazvin University of Medical Sciences, Qazvin, Iran. \\ ${ }^{6}$ Health Products Safety Research Center, Qazvin University of Medical Sciences, Qazvin, Iran. \\ $\triangle_{\text {r.mahmodi@yahoo.com }}$ \\ https://doi.org/10.34302/crpjfst/2020.12.5.14

\begin{tabular}{ll}
\hline Article history: & ABSTRACT \\
Received: & Colon cancer is an important worldwide cause of death in human which is \\
29 August 2020 & treated commonly by chemotherapy, radiotherapy and surgery methods with \\
Accepted: & different side effects. Natural treatment such as microbial cell wall extract is \\
\multicolumn{1}{c}{ 25 December 2020} & suggested to be used as an effective alternative of chemical drugs for \\
\hline Keywords: & treatment of colon cancers without any side effect. Saccharomyces boulardii \\
Probiotic Saccharomyces & is used in probiotic foods and supplement capsules in viable and yeast cell \\
boulardii; & wall extract forms. At the present study, we in vitro investigate the \\
Caco-2 cell; & anticancer properties of $S$. boulardii supernatant (SBS) on colon cancer \\
Apoptosis; & cells. We found that, SBS without dilution after 72 hours successfully killed \\
Survivin gene expression; & the colon cancer cells. Also, this treatment induced apoptosis and down- \\
Anticancer treatment. & regulated the expression of survivin gene significantly. However, effects of \\
& SBS without dilution after 24 and 48 hours were considerable. \\
& Downregulation of survivin gene expression by functional compounds in \\
& SBS induced apoptosis and killed the colon cancer cells successfully. \\
& However, future in vivo and in vitro investigation of anticancer effects of \\
& SBS on other cancer cells are suggested to be implemented. \\
\hline
\end{tabular}

\section{Introduction}

Cancer is the worldwide public health challenge and the second disease leading to death. In the year 2019, 1,762,450 new cases and 606,880 deaths because of cancer diseases were reported just in the united states. Colon cancer also is known as one of the most probable cancer disease and cause of death in human recently around the world. In the united states, 101,420 new cases and 51,020 deaths because of this cancer was reported last year (Siegel, Miller, \& Jemal, 2019). Radiotherapy, chemotherapy and surgery are methods commonly used for treatment of cancer cells and promotion of health status of the cancer patients (Garrett, 2019). Oxaliplain, leucovorin, and 5fluorouracil are commonly used as chemotherapy regimen for colon cancer treatment; however, hand numbness, tingling, dizziness, weakness and loss of muscle tone are the prominent side effects of these chemical drugs (Grothey et al., 2018). Researchers have always been studying to find efficient alternatives of strategies of cancer disease treatments because of different side effects of these drugs and methods. There are many anticancer herbal and natural drugs developed in 
recent decades to improve cancer diseases without any considerable side effects (Board, 2019). Some of these anticancer drugs are plantderived or microbial cell wall extract. Killing activity and inducing apoptosis effects of these natural drugs have been investigated and proved in vitro and in vivo for many cancer cells such as breast, colon, lung, and prostate cancer cells (Benson et al., 2017).

Saccharomyces boulardii is a yeast which has been extensively used as a probiotic with different health promotion properties in probiotic foods and supplement capsules. $S$. boulardii are employed as a beneficial yeast in viable and cell wall extract forms in foods and drugs (Altmann, 2017). S. boulardii supernatant (SBS) or cell wall extract of this yeast is produced by ultra-centrifugation of yeast biomass yielded from activated cells (Brun et al., 2017). There are many researches showed considerable antioxidant, anti-inflammatory, anticancer, and killing activities on cancer cells for SBS. Also, SBS inhibited and treated many chronological disorders such as inflammatory bowel disease, Crohn's disease, and ulcerative colitis successfully (Warila \& Hoover, 2017). However, viable form of $S$. boulardii had preventive effects on some diarrheal pathogens such as Clostridium difficile and Citrobacter rodentium. Inhibitory effect of this probiotic yeast also has been showed for fungal pathogens as well as Candida albicans (Warila \& Hoover, 2017). It is worthy to be noted that anticancer activity of SBS on some cancer cells have been investigated by some researchers and they found this treatment effective and efficient for killing cancer cells. Mannan, Beta Glucan, and bioactive peptide compounds contribute to cancer cell killing activity of SBS (Warila \& Hoover, 2017). The aim of this study was in vitro investigation of killing, inducing apoptosis and down-regulation of survivin gene expression activities of SBS on caco- 2 cell line as colon cancer cells.

\section{Materials and methods}

\subsection{Yeast cell wall extract of $S$. boulardii}

Sacharomyces boulardii was purchased from a local drug store in Qazvin, Iran as commercial lyophilized yeast in a dietary supplement capsule (®YOMOGI, Germany). The strain was mixed with DMEM culture 100 $\mathrm{mg} / \mathrm{mL}$ then incubated overnight at $37{ }^{\circ} \mathrm{C}$. Incubated yeast cells were centrifuged at 20,000 $\mathrm{g}$ for $15 \mathrm{~min}$ then the supernatant was collected. The collected supernatant was filtered through $0.22 \mu \mathrm{m}$ filters (Sigma-Alrrich, Budapest) to remove the residual yeast cells. Also, the filtered supernatant was diluted 1:2 with DMEM. Supernatants without dilution and diluted 1:2 with DMEM were used as SBS treatments for activated caco- 2 cells.

\subsection{Caco-2 cell culture and treatments}

Caco-2 cell line (Pasteur Institute, Iran) were cultured and activated in DMEM culture (Gibco, MD, USA) by incubating at $37^{\circ} \mathrm{C}$ with $5 \% \mathrm{CO} 2$. Cells were passaged once a week and subcultured into 96-well plates at $80 \%$ confluence. After formation of cell monolayer for 48 hours, cells were treated with SBS without dilution and 1:2 diluted. All treated cells were analyzed for viability, cell apoptosis and survivin gene expression after 24, 48, and 72 hours.

\subsection{MTT assay}

MTT assay (m 3-(4,5-dimethylthiazol-2-yl)2,5-diphenyltetrazolium bromide) was used to measure viability of treated cells. Caco- 2 cells were cultured in DMEM at $37{ }^{\circ} \mathrm{C}$ with $5 \% \mathrm{CO} 2$ then subcultured into 96 -well plates at $80 \%$ confluence. After formation of cell monolayer within 48 hours, cells were treated with SBS without dilution and diluted 1:2 for 24,48 , and $72 \mathrm{~h}$. For MTT assay, each well was renewed with the medium containing $0.5 \mathrm{mg} / \mathrm{mL}$ MTT then incubated for $4 \mathrm{~h}$. After incubation, medium was discarded and dimethyl sulfoxide (DMSO; Merck, Germany) was added into each well. Because of enzymatic reaction of tetrazolium MTT (yellow) to formazan (purple at $570 \mathrm{~nm}$ ) in viable cells, absorbance of each well was 
measured by microplate reader instrument model Elx808 (Bio-Tek, Vinooski VT, USA) to evaluate the viability of the treated cells. Percentage of cell viability was calculated as:

Cell viability $(\%)=\mathrm{Ae}-\mathrm{An} / \mathrm{Ac}-\mathrm{An} \times 100$

While Ae is the absorbance of the experiment group, An is the absorbance of the blank group, and Ac is the absorbance of the control group (Warila \& Hoover, 2017).

\subsection{Cell apoptosis analysis}

Annexin V-FITC and propidium iodide (PI) staining using cell apoptosis kit (Ebioscience, San Diego, USA) was used to measure and detect cell apoptosis in treated caco-2 cells. According to the manufacturer's instructions, 1 $\times 10^{6}$ cells/well seeded in 6 -well plates then treated with diluted and complete SBS for 24hour intervals until 72 hours. After treatments, cells were harvested then washed using phosphate-buffered saline (PBS) and incubated in Annexin V-FITC and PI for 30 and $5 \mathrm{~min}$ respectively at room temperature in a dark place. After staining, expression of Annexin and PI were measured employing the FACS-Calibur flow cytometer instrument (Becton, Dickinson Immunocytometry system, CA, USA).

\subsection{Measurement of survivin gene expression}

Relative expression of survivin gene was measured in treated cells using quantitative reverse transcriptase real-time PCR (qRT-PCR) method. First, total RNA of each well was extracted using tissue total RNA extraction kit (GeneAll, Korea) according to the manufacturer's instructions. Then the extracted RNA was reverse transcribed to synthesize cDNA using RT-PCR kit (GeneAll, Korea) based on manufacturer's instructions and the PCR thermal-cycler instrument ABI model 9092 (ABI, Applied biosystems, USA). Quality and quantity of the extracted RNA and the synthesized cDNA were measured using Nanodrop 2000c spectrophotometer (ThermoFisher Scientific, USA). Survivin gene expression implemented using quantitative RTPCR of synthesized cDNA. For this evaluation, $25 \mu \mathrm{L}$ reaction mixture were mixed with $12.5 \mu \mathrm{L}$
qRT-PCR SYBR green ROX master mix (Ampliqon, Denmark), $1 \mu \mathrm{L}$ of each primer $(5 \mu \mathrm{M} / \mu \mathrm{L}), 2 \mu \mathrm{L}$ of template cDNA and deionized sterilized water to $25 \mu \mathrm{L}$ as the final volume of the reaction. Primers sequences were synthesized by Sina Colon company (Sina Colon, Tehran, Iran) and used to measure the expression of survivin gene as follows: Forward; 5'-ATG GCA CGG CGC ACT TT-3' and Reverse; 5'-TCC ACT GCC CCA CTG AGA A-3 . qRT-PCRs were performed in a RotorGene real-time PCR instrument model 6000 (QiaGen, USA) with $15 \mathrm{~min}$ at $95{ }^{\circ} \mathrm{C}$ as initial denaturation, followed by 40 cycles including $15 \mathrm{~s}$ at $95^{\circ} \mathrm{C}$ and $1 \mathrm{~min}$ at $60{ }^{\circ} \mathrm{C}$. After determining cycle threshold (CT) for each reaction, the relative quantification of survivin gene expression was measured as previously described by Motawi et al. (2014) (Motawi, Bustanji, EL-Maraghy, Taha, \& Al-Ghussein, 2014).

\subsection{Statistical analysis}

One-way analysis of variance (ANOVA) was used to measure significant $(\mathrm{P}<0.05)$ differences among the groups of data employing SPSS software version 22 (SPSS Inc., Chicago, IL, USA). All measurement and experiments were done in triplicates.

\section{Results and discussions}

Effects of SBS (without dilution and diluted $1: 2 \mathrm{w} / \mathrm{w}$ ) for different duration treatment on viability of colon cancer cell line, caco-2, were investigated using MTT assay (FIGURE 1). As it is shown in FIGURE 1, treatment after 48 and 72 hours with SBS without dilution significantly more killed the caco-2 cells. However, the killing activity of the treatment with SBS without dilution after 24 hours was considerable, but the killing activity of this treatment was significantly lower than that of other duration treatments of without diluted SBS. Consequently, in vitro cytotoxic activity of SBS on caco-2 cells are shown at the present study. However, dilution of SBS significantly affects the killing activity of this treatment. 
FIGURE 2 illustrates analysis of flow cytometry of the caco-2 cells treated with 1:2 diluted (A) and without dilution (B: 24, C: 48, and D: 72 hours) SBS. The proportion of viable, early apoptotic and late apoptotic/necrotic cells are shown in Q4, Q3, and Q2 quadrants respectively. The proportions of apoptotic cells, including early and late apoptosis, for caco-2 cells treated with 1:2 diluted SBS was 3.75. However, these proportions for cells treated with SBS without dilution after 24, 48, and 72 hours were measured 18.28, 12.92, and 53.2\% respectively. Also, the percentage of viable cells in caco-2 cells treated without dilution after 72 hours $(\mathrm{Q} 4=37.6 \%)$ was significantly lower than that in cells treated with other treatments. Thus, these results indicate that treatment with SBS without dilution after 72 hours induced significant apoptosis in caco-2 cells and decreased cell viability in comparison with other treatments. However, SBS treatment without dilution after 24 and 48 hours induced apoptosis (18.28 and $12.92 \%$ respectively) considerably higher than all 1:2 diluted treatment.

Caco-2 cells treated with diluted and without dilution SBS were subjected to analysis of survivin gene expression (all synthesized cDNA was quantitively and qualitatively appropriate for qRT-PCR) by qRT-PCR and the results are illustrated in FIGURE 3. Similar to results of MTT assay and apoptotic analysis of treated cells, treatment with SBS without dilution after 72 hours decreased the survivin gene expression significantly higher than other treatments as can be seen in FIGURE 4. Also, other duration times of without dilution treatments were more considerably effective than 1:2 diluted treatments in reduction of survivin expression. Inducing apoptosis decrease the survivin expression which shows lower cell viability in MTT assay. As a result of the study, treatment with SBS without dilution after 72 hours successfully killed the colon cancer cells (caco2 cell line). This killing activity was proved by investigation of inducing apoptosis, decreasing cell viability, and survivin expression in the treated cells.
At the present study, we investigated killing activity of $S$. boulardii supernatant or SBS without dilution and diluted 1:2 on viability of colon cancer cells (caco-2). The results indicated that SBS without dilution after 72 hours significantly killed more caco- 2 cells than other treatments; consequently, this treatment can be considered as an efficient and new colon cancer cell killer which is studied in vitro for caco-2 cells.

Yeast cell wall extract consists of many bioactive compounds with different activities including antifungal, antioxidant, immunomodulating, anti-inflammatory, and anticancer activities. Also, cell wall extract or supernatant of $S$. boulardii biomass as a probiotic yeast showed many functional and bioactive properties as mentioned previously (Datta, Timson, \& Annapure, 2017). It is previously found that Mannan and Beta Glucan compounds presenting in cell wall extract of $S$. boulardii and other yeast cells usually kill the tumor cells and inhibit the growth effectively. However, these polysaccharides as well as Beta Glucan with antitumor activity was extracted from other sources such as some fruit and vegetables (Suryavanshi et al., 2013). Also, a varied set of complex phenolic compounds present in SBS which are associated with the antioxidant and anticancer activity of $S$. boulardii cell wall extract. There is a strength association between antioxidant and anticancer activity of a bioactive compound (Ryan et al., 2011). Gallic acid and catechin are the most prevalent phenolic compounds in SBS. Therefore, a complex interaction of antioxidant compounds (phenolic acids) and polysaccharides (mannan and beta glucan) explain killing activity of cancer cells for SBS. However, it is reported that these compounds are heat stable with less than $1 \mathrm{KD}$ molecular weight (Murzyn, Krasowska, Stefanowicz, Dziadkowiec, \& Łukaszewicz, 2010).

We found that SBS after 72 hours induce apoptosis in caco-2 cells. Probiotic supernatant and cell wall extract up-regulate the expression of apoptosis gene in cancer cells. Nouzari et al. (2019) have shown that supernatant of 
Lactobacillus paracasei biomass as a probiotic bacterium induced the apoptosis regulation in caco-2 cells which decreased the viability of these cells effectively (Nozari et al., 2019). Shyu et al. (2014) also reported that probiotic supernatant significantly increases gene expression regulation of apoptosis in HCT116 and HT29 cell lines (Shyu, Oyong, \& Cabrera, 2014). Huang et al. (2016) illustrated anticancer effect of Lactobacillus species supernatant on HT29 cells. They extracted proteins from supernatant of probiotic bacteria and showed a strong association between cancer cell killing activity of cell wall extract and the protein content (Huang et al., 2016). However, there are many studies showed anticancer effect of bioactive peptides presented in protein content of the cell wall extracts. Also, it is proved that the protein fraction of the probiotic supernatant induces apoptosis and contribute to death in cancer cells. This protein fraction affects the signaling pathway of apoptosis in carcinoma colon cells (Naimah, Al-Manhel, \& Al-Shawi, 2018).

Survivin gene is categorized in a unique group of protein recently described as inhibitors of apoptosis (IAP) which inhibit the induced apoptosis in cell. Survivin expresses in mitosis step in the cell cycle, also expression of this gene is inhibited and reduced during the apoptosis ( $\mathrm{Li}$ et al., 2018). Consequently; every treatment reducing the expression of survivin gene induces and increases the apoptosis in cell (Arafat et al., 2017). At the present study, SBS without dilution treatment after 72 hours decreased the survivin gene expression in caco-2 cells therefore killed them successfully. Schwab et al. (2009) showed that butyrate and mesalazine down-regulated and decreased expression of IAP proteins such as survivin gene in caco-2 cells successfully. They indicated that downregulation of the survivin gene inducing apoptosis in caco- 2 cells is caused by interaction of butyrate and mesalazine with IAP proteins such as survivin (Schwab, Reynders, Steinhilber, \& Stein, 2009). Hwang et al. (2015) also indicated decreasing effect of Shogaol, a fraction if ginger plant, on survivin gene expression as an antiapoptotic protein in caco-2 cells. It could be considered that the main reason of colon cancer killing activity of SBS is probably because of down-regulation effect of this treatment on expression of IAP protein as well as survivin in caco-2 cells (Hwang, Lee, Oh, Lee, \& Kwon, 2015). There are some researches about the effects of SBS on signaling and growth rate of many cancer cells; however, the effects of SBS on caco- 2 cells have not been investigated previously and now it is studied at the present study. Most of researchers believe that Beta Glucan and some proteins in yeast cell wall extract describe the killing activity of SBS on caco-2 cells (Fortin, Aguilar-Uscanga, Vu, Salmieri, \& Lacroix, 2018). Cancer treatment activity of some drugs such as naproxen and cromolyn also is associated with reducing surviving gene expression in cancer cells as well as caco-2 cell line (Henry, D'hondt, Andre, Holemans, \& Canon, 2004). In addition to antiinflammatory and antioxidant activities of SBS, cancer treatment function of yeast cell wall extract of probiotic $S$. boulardii is suggested to be employed by some researchers recently (Chen et al., 2009). Fortin et al. (2018) investigated preventive effect of cell wall extract of $S$. boulardii in vivo on colon cancer in rats treated with Dimethylhydrazine. They found that SBS successfully prevented and treated the colon cancer in rats (Fortin et al., 2018). Fatemi et al. (2019) also demonstrated inhibitory effect of SBS on breast cancer induced by Dimethylbenza-Anthracene in rats. They found that SBS reduced the tumor size and spread of breast cancer cells by inducing apoptosis (Fatemi, Ghandhari, \& Karimi, 2019). At the present study, we in vitro killed the colon cancer cells by SBS without dilution treatment after 72 hours. We found that SBS down-regulated the expression of survivin gene and induced apoptosis to kill the caco- 2 cells successfully. 


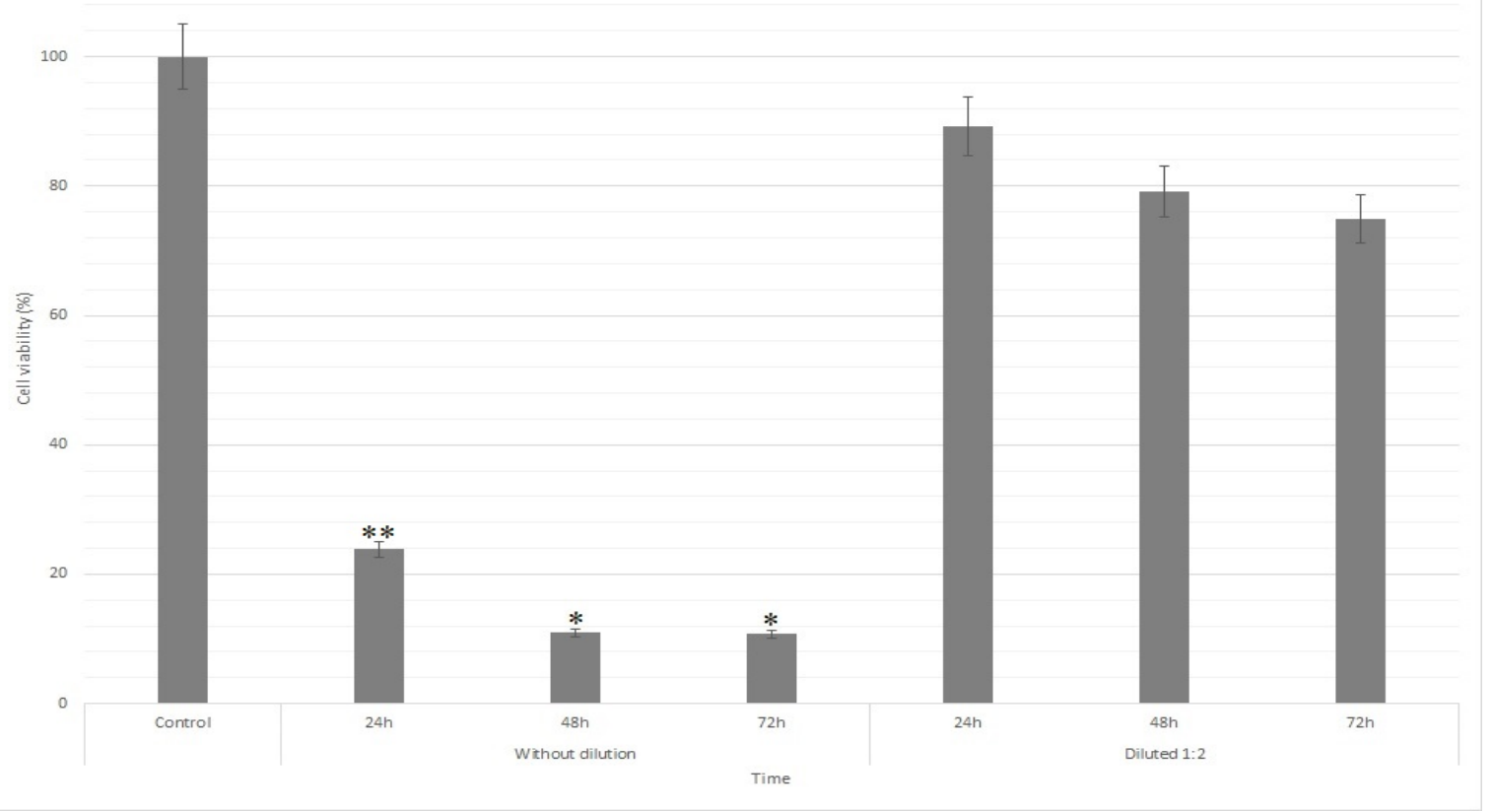

Figure 1. Cytotoxicity determination of SBS on Caco-2 cells by MTT assay.

$*$ and $* *$ indicates significant difference
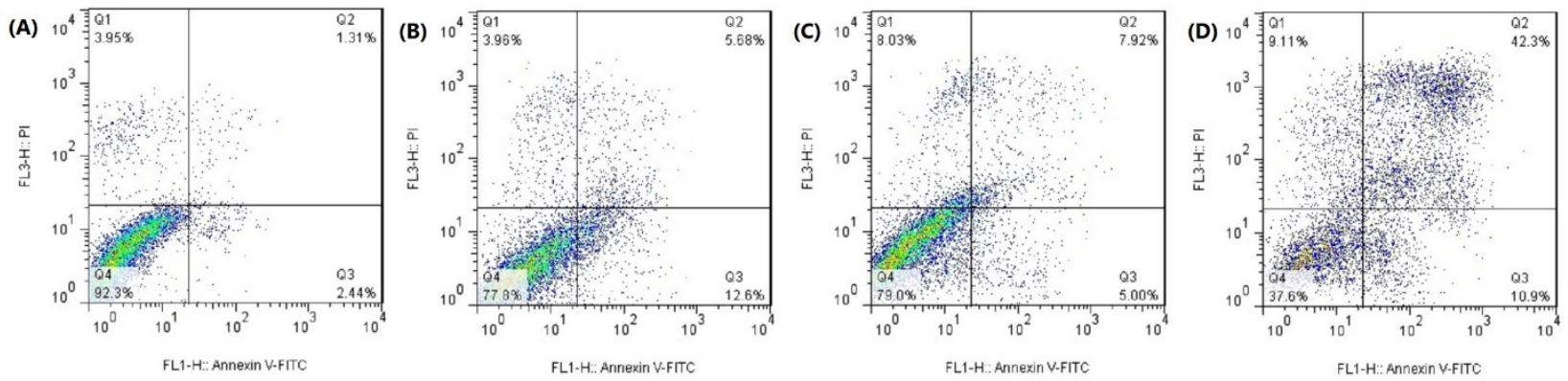

Figure 2. Analysis of SBS (Diluted 1:2 (A); and without dilution for 24h (B), 48h (C), and 72h (D)) on Caco-2 cells with apoptosis determination by flow cytometry.

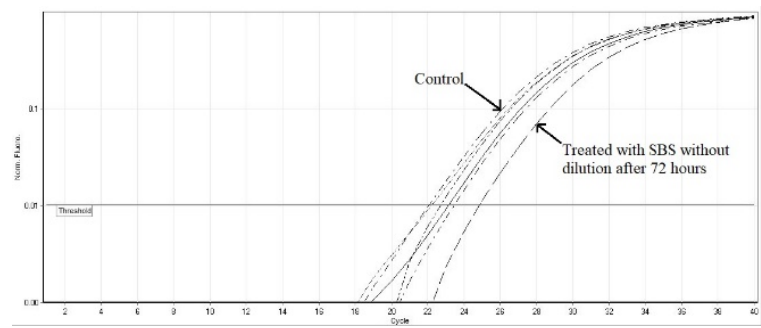

Figure 3. Normalized fluorescence curves of qRT-PCR of survivin gene mRNA for treated caco-2 cells with SBS. 


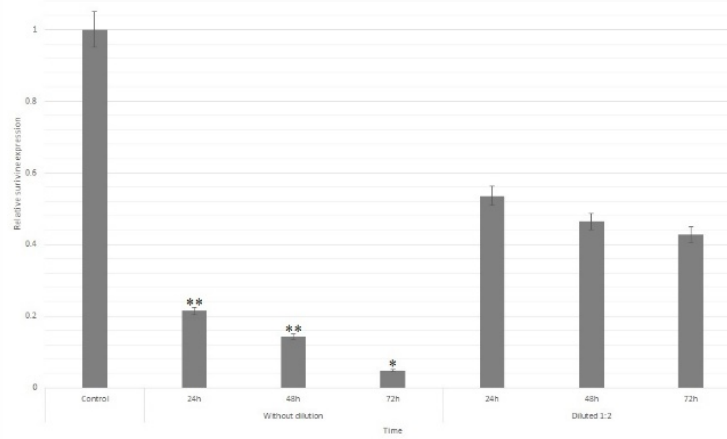

Figure 4. Relative Survivine gene expression in Caco-2 cells treated with SBS. $*$ and $* *$ indicates significant difference

\section{Conclusions}

At the present study, we investigated the killing activity and in vitro anticancer properties of yeast cell wall extract of $S$. boulardii on colon cancer (caco-2) cells. We found that SBS without dilution significantly killed caco-2 cells after 72 hours; however, the effect of this treatment after 24 and 48 hours were considerable. SBS without dilution treatment after 72 hours reduced the survivin expression in caco-2 cells and induced 53.2\% apoptosis. Functional compounds such as Beta Glucan, Mannan and bioactive peptides in protein fraction of SBS contribute to inhibitory effect on survivin gene expression and against caco- 2 cell viability. We strongly suggest SBS to be employed as a colon cancer treatment; however, further in vivo investigations and studying the killing activity of colon cancer cells with cell wall extract of other yeasts are necessary to be implemented consequently.

\section{References}

Altmann, M. (2017). The Benefits of Saccharomyces Boulardii. In The Yeast Role in Medical Applications: IntechOpen.

Arafat, W., El Ashry, M., Abd Alrazek, M., Matta, C., El Aleem, E., Kamel, E., . . . Samir, M. (2017). The relation between survivin gene expression and urinary bladder cancer disease. Hospice and Palliative Medicine International Journal, 1(2).

Benson, A. B., Venook, A. P., Cederquist, L., Chan, E., Chen, Y.-J., Cooper, H. S., . . .
Fichera, A. (2017). Colon cancer, version 1.2017, NCCN clinical practice guidelines in oncology. Journal of the National Comprehensive Cancer Network, 15(3), 370-398.

Board, P. A. T. E. (2019). Colon Cancer Treatment (PDQ $\left.{ }^{\circledR}\right)$. In PDQ Cancer Information Summaries [Internet]: National Cancer Institute (US).

Brun, P., Scarpa, M., Marchiori, C., Sarasin, G., Caputi, V., Porzionato, A., . . Castagliuolo, I. (2017). Saccharomyces boulardii CNCM I-745 supplementation reduces gastrointestinal dysfunction in an animal model of IBS. PloS one, 12(7).

Chen, X., Fruehauf, J., Goldsmith, J. D., Xu, H., Katchar, K. K., Koon, H. W., . . . Kelly, C. P. (2009). Saccharomyces boulardii inhibits EGF receptor signaling and intestinal tumor growth in Apcmin mice. Gastroenterology, 137(3), 914-923.

Datta, S., Timson, D. J., \& Annapure, U. S. (2017). Antioxidant properties and global metabolite screening of the probiotic yeast Saccharomyces cerevisiae var. boulardii. Journal of the Science of Food and Agriculture, 97(9), 3039-3049.

Fatemi, M., Ghandhari, F., \& Karimi, N. (2019). Effects of the Cell Debris and Supernatant of Saccharomyces boulardii on 7, 12Dimethylbenz (a) Anthracene-Induced Breast Cancer in Rats. Journal of Kermanshah University of Medical Sciences(In Press). 
Fortin, O., Aguilar-Uscanga, B., Vu, K. D., Salmieri, S., \& Lacroix, M. (2018). Cancer chemopreventive, antiproliferative, and superoxide anion scavenging properties of Kluyveromyces marxianus and Saccharomyces cerevisiae var. boulardii cell wall components. Nutrition and cancer, 70(1), 83-96.

Garrett, W. S. (2019). The gut microbiota and colon cancer. Science, 364(6446), 11331135.

Grothey, A., Sobrero, A. F., Shields, A. F., Yoshino, T., Paul, J., Taieb, J., . . Labianca, R. (2018). Duration of adjuvant chemotherapy for stage III colon cancer. New England Journal of Medicine, 378(13), 1177-1188.

Henry, S., D'hondt, L., Andre, M., Holemans, X., \& Canon, J. (2004). Saccharomyces cerevisiae fungemia in a head and neck cancer patient: a case report and review of the literature. Acta Clinica Belgica, 59(4), 220-222.

Huang, L., Shan, Y.-J., He, C.-X., Ren, M.-H., Tian, P.-J., \& Song, W. (2016). Effects of L. paracasei subp. paracasei X12 on cell cycle of colon cancer HT-29 cells and regulation of mTOR signalling pathway. Journal of functional foods, 21, 431-439.

Hwang, J. S., Lee, H.-C., Oh, S. C., Lee, D.-H., \& Kwon, K. H. (2015). Shogaol overcomes TRAIL resistance in colon cancer cells via inhibiting of survivin. Tumor Biology, 36(11), 8819-8829.

Li, J., Han, Y., Zhou, D., Zhou, Y., Ye, M., Wang, H., \& Du, Z. (2018). Downregulation of Survivin gene expression affects ionizing radiation resistance of human T98 glioma cells. Cellular and molecular neurobiology, 38(4), 861-868.

Motawi, T. M., Bustanji, Y., EL-Maraghy, S., Taha, M. O., \& Al-Ghussein, M. A. (2014). Evaluation of naproxen and cromolyn activities against cancer cells viability, proliferation, apoptosis, p53 and gene expression of survivin and caspase-3. Journal of enzyme inhibition and medicinal chemistry, 29(2), 153-161.
Murzyn, A., Krasowska, A., Stefanowicz, P., Dziadkowiec, D., \& Łukaszewicz, M. (2010). Capric acid secreted by $S$. boulardii inhibits C. albicans filamentous growth, adhesion and biofilm formation. PloS one, 5(8).

Naimah, A. K., Al-Manhel, A. J. A., \& AlShawi, M. J. (2018). Isolation, purification and characterization of antimicrobial peptides produced from Saccharomyces boulardii. International Journal of Peptide Research and Therapeutics, 24(3), 455-461.

Nozari, S., Faridvand, Y., Etesami, A., Ahmad Khan Beiki, M., Miresmaeili Mazrakhondi, S. A., \& Abdolalizadeh, J. (2019). Potential anticancer effects of cell wall protein fractions from Lactobacillus paracasei on human intestinal Caco- 2 cell line. Letters in applied microbiology, 69(3), 148-154.

Ryan, E. P., Heuberger, A. L., Weir, T. L., Barnett, B., Broeckling, C. D., \& Prenni, J. E. (2011). Rice bran fermented with Saccharomyces boulardii generates novel metabolite profiles with bioactivity. Journal of agricultural and food chemistry, 59(5), 1862-1870.

Schwab, M., Reynders, V., Steinhilber, D., \& Stein, J. (2009). Combined treatment of Caco-2 cells with butyrate and mesalazine inhibits cell proliferation and reduces Survivin protein level. Cancer letters, 273(1), 98-106.

Shyu, P. T., Oyong, G. G., \& Cabrera, E. C. (2014). Cytotoxicity of probiotics from Philippine commercial dairy products on cancer cells and the effect on expression of cfos and cjun early apoptotic-promoting genes and interleukin- $1 \beta$ and tumor necrosis factor- $\alpha$ proinflammatory cytokine genes. BioMed research international, 2014.

Siegel, R. L., Miller, K. D., \& Jemal, A. (2019). Cancer statistics, 2019. CA: a cancer journal for clinicians, 69(1), 7-34.

Suryavanshi, A., Agarwal, A., Kaler, A., Bihade, U., Kaur, J., Tikoo, K. B., \& Banerjee, U. C. (2013). Comparative studies on the antioxidant potential of vanillinproducing Saccharomyces boulardii 
extracts. Oxidants and Antioxidants in Medical Science, 2(3), 201-209.

Warila, R., \& Hoover, R. (2017). The role of Saccharomyces boulardii in the treatment of refractory recurrent Clostridium difficile infection. International Journal of Food and Allied Sciences, 3(1), 20-26. 